\title{
Developing an Analytical Framework to Characterize Student Reasoning about Complex Processes
}

\author{
Emily E. Scott, ${ }^{\text {* }}$ Charles W. Anderson, ${ }^{\dagger}$ K. K. Mashood, ${ }^{\sharp \S}$ Rebecca L. Matz," \\ Sonia M. Underwood, ${ }^{\neq \pi}$ and Vashti Sawtelle ${ }^{\sharp \#}$ \\ 'Department of Teacher Education, "Department of Physics and Astronomy, and "Hub for \\ Innovation in Learning and Technology, Michigan State University, East Lansing, MI 48824; \\ ҒSTEM Transformation Institute and 'Department of Chemistry and Biochemistry, Florida \\ International University, Miami, FL 33199; " Lyman Briggs College, Michigan State University, \\ East Lansing, MI 48824
}

\begin{abstract}
Real-world processes are complex and require ideas from multiple disciplines to be explained. However, many science courses offer limited opportunities for students to synthesize scientific ideas into coherent explanations. In this study, we investigated how students constructed causal explanations of complex phenomena to better understand the ways they approach this practice. We interviewed 12 undergraduate science majors and asked them to explain real-world phenomena. From these interviews, we developed a characterization framework that described the reasoning patterns we found. In this framework, we identified three explanatory frames that differentiated the kinds of explanations students provided: a colloquial frame, wherein participants activated conceptual resources based on personal experience using everyday language; an emerging mechanistic frame, wherein participants used scientific concepts in semicoherent ways; and a causal mechanistic frame, wherein participants cohesively drew upon scientific conceptual resources to construct mechanistic explanations. Overall, the causal mechanistic frame was the least prevalent frame invoked by students. Instead, many drew on an emerging mechanistic frame and struggled to identify and apply scientific concepts to real-world scenarios. We advocate for incorporating opportunities to reason about real-world phenomena into undergraduate science curricula to provide students with experience integrating scientific concepts to explain real-world phenomena.
\end{abstract}

\section{INTRODUCTION}

Real-world phenomena are complex in nature and require solutions that span different disciplines in the natural sciences (Hmelo-Silver and Azevedo, 2006; Jacobson and Wilensky, 2006). Consequently, it is critical that science educators help students learn to reason across traditional disciplinary boundaries (McNeill and Krajcik, 2008; American Association for the Advancement of Science [AAAS] 2011; National Research Council [NRC], 2012a). To do this, reformers advocate for approaching science instruction from a three-dimensional perspective built on 1) core disciplinary ideas of different science disciplines, 2) crosscutting concepts that are foundational across science disciplines, and 3) scientific practices that describe how scientific knowledge is generated and used (AAAS, 2011; NRC, 2012b). However, implementing this three-dimensional approach is challenging, especially at the undergraduate level, where curricula are usually organized into discipline-specific courses that rely on a few idealized phenomena (e.g., projectiles in a vacuum, reactions between pure chemical substances) designed to exemplify concepts in a single discipline (Sharma and Anderson, 2009; Gunckel, 2010). As a result, science courses tend to provide
Hannah Sevian, Monitoring Editor Submitted Oct 31, 2017; Revised May 14, 2018; Accepted May 17, 2018

CBE Life Sci Educ September 1, 2018 17:ar49 DOI: $10.1187 /$ cbe.17-10-0225

*Address correspondence to: Emily E. Scott (scottemi@uw.edu).

(c) 2018 E. E. Scott et al. CBE-Life Sciences Education @ 2018 The American Society for Cell Biology. This article is distributed by The American Society for Cell Biology under license from the author(s). It is available to the public under an Attribution-Noncommercial-Share Alike 3.0 Unported Creative Commons License (http:// creativecommons.org/licenses/by-nc-sa/3.0). "ASCB®" and "The American Society for Cell Biology ${ }^{\prime}$ " are registered trademarks of The American Society for Cell Biology. 
students with few opportunities to confront complex processes that require the synthesis of ideas from different scientific fields to form coherent, scientific explanations (Stevens et al., 2005; Ledford, 2015).

To address this limitation, reformers have advocated that universities incorporate learning experiences into their science programs that afford students opportunities to reason about complex processes and synthesize multiple scientific ideas from different fields (Jacobson and Wilensky, 2006; AAAS, 2011; NRC, 2012b; Cooper et al., 2015). These calls highlight the need for understanding how students currently make sense of complex processes to inform the kinds of instructional approaches best suited for achieving this goal. To address this need, we conducted a study to investigate how undergraduate science students explain complex, real-world processes that they may encounter outside science courses.

Our analyses draw on two bodies of research about the practice of constructing explanations. The first draws from literature on how students' explanatory frames for explaining complex, everyday processes influence the kinds of conceptual resources they activate when constructing their accounts. In other words, we are interested in the different ways students activate and synthesize both their scientific and everyday knowledge to explain complex processes. We define an "explanatory frame" after Hammer et al. (2005) as the "set of expectations an individual has about the situation in which she finds herself that affect what she notices and how she thinks to act" (p. 9). The second body of research compares and contrasts the conceptual resources that students activate during their reasoning with the key features of a particular kind of explanatory approach: causal mechanistic explanations. While there is extensive work conducted in both of these areas by other researchers, we did not find studies that examined how students' framing of an activity related to their ability to produce causal mechanistic explanations. Therefore, our work provides a novel synthesis of these two research lines to better understand how undergraduate science majors construct explanations about scientific processes.

\section{Conceptual Resources and Explanatory Frames}

During the course of undergraduate science majors' science education, students are exposed to a plethora of ideas that originate from scientific disciplines, such as "one gene-one polypeptide" from biology, "matter must be conserved in closed systems" from physics, and "like substances are soluble" from chemistry. These scientific ideas, along with others from students' personal experiences, are conceptual resources that they can draw on when reasoning about the natural world (Redish, 2004; Hammer et al., 2005; Scherr and Hammer, 2009; Keil, 2012). Conceptual resources are the fine-grained pieces of knowledge that students activate and synthesize in real time to reason about a particular context; however, their activation in the context of a particular phenomenon may be more or less appropriate for explaining how or why that phenomenon occurs (Hammer et al., 2005). We draw on the idea of conceptual resources in our research because when students construct explanations about complex, real-world processes, they activate and synthesize conceptual resources they have compiled from both their science courses and personal life experiences in order to productively explain the phenomena (Shtulman and Valcarcel, 2012).
Students activate particular conceptual resources depending on their interpretation of the purpose of an activity in which they engage; in other words, the knowledge they access will depend on how they interpret the framing of an activity (Hammer and Elby, 2002; Hammer et al., 2005; Redish, 2004). Frames are constructed based on students' past experiences and their interpretations of what is required in the setting in which they find themselves (Redish, 2004). Students may subconsciously consider multiple frames when deciding what knowledge is relevant during an activity, demonstrating the dynamic nature of students' reasoning with respect to knowledge (Scherr and Hammer, 2009; Berland and Hammer, 2012). When students activate a particular explanatory frame, Hammer et al. (2005) suggest that they are activating a "locally coherent set of resources" that form a cognitive unit particular to their immediate situation. One example of an explanatory frame is students' use of colloquial language to explain phenomena in a narrative or scripted way, often drawing on everyday occurrences for support in their explanation (Chi et al., 2012). Another example of an explanatory frame is students' use of scientific language to convey ideas that originate from scientific disciplines in a manner consistent with experts in those fields (Carey, 1986).

A primary goal of science educators is to help students learn to appropriately activate conceptual resources and explanatory frames in order to foster a deep understanding of science (Hammer et al., 2005; NRC, 2012b). One approach for doing this is outlined in the Framework for K-12 Science Education, which describes a coherent set of conceptual resources that students can use to achieve this goal (NRC, 2012b). Specifically, it suggests that, when students activate and synthesize conceptual resources about the core disciplinary ideas of scientific fields, crosscutting concepts that are fundamental across science fields, and scientific practices that generate knowledge, they are able to reason "less like novices and more like experts" who "understand the core principles and theoretical constructs of their field" and "use them to ... tackle novel problems" (NRC, 2012b, p. 25). However, these conceptual resources facilitate a deep understanding of science only when students adopt an explanatory frame that synthesizes and arranges these resources in coherent ways that align with scientific norms (Clark and Linn, 2003).

\section{Study Phenomena}

In this study, we asked students to construct explanations about several complex, real-world processes in biological systems in order to investigate the kinds of explanatory frames-and the associated conceptual resources-that students invoked when engaging in this activity. We deliberately chose processes that students may have experienced to provide them with opportunities to activate explanatory frames and conceptual resources from multiple spheres of life when creating their accounts; we avoided processes that occur across large scales (e.g., evolution, climate change) that students may only abstractly conceive (Jacobson and Wilensky, 2006). Consequently, our processes were constrained both temporally and spatially, meaning they occurred in local settings over the course of several minutes to a couple of weeks. However, these processes were also scientifically complex, in that they required students to activate and synthesize knowledge from multiple scientific fields to provide a full explanation (i.e., all of our processes were situated in biological systems for which an explanation that incorporated 
ideas from chemistry and physics was necessary to construct a causal mechanistic explanation). The processes we chose were

1. Why does a blister form when a person touches a hot pan? (Heatburn)

2. Why does an egg white go from a runny substance to a solid when it is boiled? (Egg)

3. How can someone get sick from another person sneezing nearby? (Sneeze)

4. How does an ultrasound wand applied to the outside of a person's body produce an image from inside the body? (Ultrasound)

5. How does an actor build muscle? (Protein)

\section{Causal Mechanistic Explanations}

We focused our research on the scientific practice of constructing explanations, because explaining how and why phenomena occur is both a primary goal of scientific endeavors (AAAS, 1993; Chi et al., 2012; NRC, 2012b) and a key indicator of students' understanding of scientific ideas (Windschitl et al., 2008). There are a variety of types of explanations that scientists employ to communicate their ideas, like covering law explanations, which are explanations that describe events as logical outcomes of natural processes expressed as laws (e.g., the ideal gas law); statistical-probabilistic explanations, which focus on patterns and trends in data; and causal mechanistic explanations, which use patterns in data to elucidate the underlying mechanisms occurring in a system (Russ et al., 2009; Braaten and Windschitl, 2011; Lombrozo and Vasilyeva, 2017). The different kinds of explanations have affordances and limitations around the information they convey (Braaten and Windschitl, 2011; Weinrich and Talanquer, 2015). In this study, we focused solely on whether or not students constructed causal mechanistic explanations, because such explanations have high explanatory power (Russ et al., 2008; Weinrich and Talanquer, 2015), are mechanistic in nature (Braaten and Windschitl, 2011; Chi et al., 2012; Passmore et al., 2013), and demonstrate a deep understanding of how and why scientific processes occur (NRC, 2012b; Cooper et al., 2016). We also knew that our pool of research participants had been exposed to relevant conceptual resources for causal mechanistic explanations of the processes we selected during their undergraduate science education.

To construct causal mechanistic explanations, students must activate conceptual resources that 1) account for multiscale processes (Chi et al., 2012), 2) are grounded in scientific ideas that describe the nature of material interactions (Russ et al., 2008; Braaten and Windschitl, 2011), and 3) apply relevant ontologies (i.e., categorization schemes) that help explain why events occur as they do (Chi et al., 1994; Gupta et al., 2010). However, this is a challenging exercise for students, who often activate alternative, nonscientific conceptual resources when explaining phenomena (Mohan et al., 2009; Chi et al., 2012; Weinrich and Talanquer, 2015). In the following section, we discuss these characteristics of causal mechanistic explanations and review literature contrasting how scientists and students activate conceptual resources around them.

Multiscale Processes. When scientists construct causal mechanistic explanations about observable phenomena, those explanations are predicated on an understanding that systems are composed of multiple scales, wherein interactions that occur in subsystems, such as atomic-molecular interactions or cellular dynamics, 1) behave differently from observable macroscale processes, 2) are often undirected in nature, and 3) result in emergent patterns at macroscopic scales (Chi et al., 2012; NRC, 2012b; Weinrich and Talanquer, 2015; Southard et al., 2017). However, when students construct causal mechanistic explanations about macroscale processes, they often ignore subscale processes in favor of describing patterns they can directly observe (Hesse and Anderson, 1992; Mohan et al., 2009; Chi et al., 2012; Weinrich and Talanquer, 2015). Therefore, we can gauge whether or not students recognize the multiscale processes consistent with a scientific explanatory approach by evaluating the features of a phenomenon (e.g., atoms, microscopic materials, observable materials) they privilege in their explanations to account for what is happening.

Grounding in Scientific Ideas. Causal mechanistic explanations make sense of phenomena based on scientific ideas (NRC, 2012b). Students can include scientific ideas in their accounts in two ways: by using foundational scientific principles to either implicitly or explicitly constrain their explanations of why phenomena occur and/or by using disciplinary ideas that are specific to the phenomena they are describing (NRC, 2012b). However, students may bring a variety of nonscientific ideas into their accounts to explain why or how phenomena occur. For example, students may use force-dynamic reasoning to explain how organisms or objects experience forces or other factors that either assist or prevent their achieving a natural tendency (Talmy, 1988; Pinker, 2007; Mohan et al., 2009; Jin and Anderson, 2012; Weinrich and Talanquer, 2015). When using force-dynamic reasoning, students may describe the events in a narrative or scripted style, usually with an initiating event that triggers other processes in an intentional, logical, sequential progression that culminates in an end goal (Chi et al., 2012; Sevian and Talanquer, 2014). Students may also ascribe teleological motivations to objects or organisms (Coley and Tanner, 2012; Weinrich and Talanquer, 2015). As students try to include scientific ideas in their explanations for why phenomena occur, they may rely on covering law relationships that make use of scientific laws or informal heuristics without understanding why those relationships occur (Maeyer and Talanquer, 2010; Braaten and Windschitl, 2011; Cooper et al., 2013). By evaluating the degree to which students activate conceptual resources that are grounded in scientific ideas when constructing causal mechanistic explanations, we gain a sense of whether or not their explanatory frames are derived from scientific roots or an alternative way of knowing.

Relevant Ontological Categorization. Scientists group materials and processes into hierarchical categories, or ontologies, that describe how similar or distinct they are (Chi et al., 1994; Gupta et al., 2010). This is done either explicitly, as with phylogenies, or implicitly when relating the materials or processes of a system with those of other systems. When ontologies are based on superficial similarities, for example likening the process of water freezing with a boiling egg solidifying, they conceal fundamentally different subscale interactions (Chi et al., 1994; Chi, 2013). Conversely, when ontologies are based on subscale mechanistic processes that appear distinct at observable scales (e.g., likening decay, animal metabolism, and 
combustion, which all involve the oxidation of organic carbon), they reveal a more cohesive view of scientific processes (Mohan et al., 2009; Jin and Anderson, 2012). Therefore, students' explanations for how and why phenomena occur depend on their decisions about what "kind" of phenomenon they are explaining. By evaluating students' ontologies, we can gain insight into how they choose the explanatory frames and conceptual resources that they activate.

One way to gain insight into students' ontological choices is by evaluating the kinds of analogies they invoke during their reasoning. We can leverage students' analogies to evaluate their ontological choices, because analogical reasoning involves relating information from an established base of knowledge about a system to an unfamiliar system to be explained (Vosniadou and Ortony, 1989). The way that students relate an analogue to an unfamiliar system can provide insight into which features of the unfamiliar system they perceive are most relevant to explaining it, such as whether they focus on subscale mechanistic similarities (e.g., common molecular interactions) or surface-level similarities (e.g., color, shape; Gentner, 1989; Vosniadou, 1989). The level at which students identify similarities tends to deepen as they acquire more knowledge (Vosniadou, 1989; Mozzer and Justi, 2012), leading to notable differences in the kinds of structures that experienced and novice learners privilege when deciding what systems are similar or not (Chi et al., 1981).

\section{Research Questions}

Our goal in this project was to discern ways that undergraduate students are able to reason about complex, real-world processes after experiencing several undergraduate science courses. We suggest that, when students construct explanations primarily using the observable properties of systems, force-dynamic or conventional wisdom-type rationales, and ontological categorization schemes based on observable features, they are invoking an explanatory frame that activates conceptual resources derived primarily from personal experiences (e.g., visual observations; Chi et al., 2012). Alternatively, when students focus their explanations on the features of subsystems, ground them in scientific ideas, and use ontologies based on the fundamental properties of a system, we suggest they are invoking an explanatory frame that activates conceptual resources more in line with scientific ideas. However, the degree of alignment depends on the coherence of the synthesis of these conceptual resources.
We use the following questions to guide our research:

1. What kinds of explanatory frames and conceptual resources do students invoke when constructing causal explanations for complex, real-world phenomena?

2. How do students' explanatory frames vary as they reason about different phenomena?

\section{METHODS \\ Data Collection}

We conducted semistructured interviews with undergraduate science majors enrolled at a large midwestern research university to better understand how they construct causal explanations about complex, real-world phenomena. This approach allowed us the flexibility to stray from our standardized protocol depending on the ideas participants offered when reasoning about a particular phenomenon (Ginsburg, 1997; Rubin and Rubin, 2005).

Because students may interpret the purpose of an interview differently from the interviewer, which can influence the kinds of reasoning and knowledge they subsequently provide (Russ et al., 2012), the participants were initially advised that one of the primary objectives of the project was to better understand how students make connections between the fields of biology, chemistry, and physics. Therefore, we suggest the participants were aware that we were interested in their use of scientific ideas from a variety of disciplines. However, we also invited students to draw upon "everyday thinking" during their reasoning. This helped ensure that all students would have ideas they could discuss that related to our phenomena, even if they were unable to invoke scientific conceptual resources for a particular phenomenon.

We solicited student participation from a second-semester physics course, because these students had taken at least one semester each of introductory chemistry, biology, and physics, ensuring they had been exposed to a range of ideas from multiple disciplines that they could potentially draw upon when constructing explanations about our phenomena. Twelve students were available to participate during our time frame at the beginning of the Fall 2015 semester and were compensated with extra credit points toward their physics course (see Table 1 for a description of participant demographics). All participants signed informed consent forms. We use pseudonyms to identify our interview participants.

Each participant was presented with a range of three to five phenomena during the course of a 60-minute interview,

TABLE 1. Demographics of undergraduate interview participants

\begin{tabular}{|c|c|c|c|c|}
\hline Pseudonym & Major & Year in college & Gender & Race \\
\hline Brad & Human biology & Senior & $\mathrm{M}$ & White/non-Hispanic \\
\hline Celeste & Kinesiology & Junior & F & White/non-Hispanic \\
\hline Daniel & Physiology & Junior & M & White/Hispanic \\
\hline Jennifer & Human biology & Junior & F & White/non-Hispanic \\
\hline Julie & Microbiology & Junior & F & Asian \\
\hline Natalie & Human biology & Junior & F & White/non-Hispanic \\
\hline Rudee & Neuroscience & Junior & $\mathrm{F}$ & White/non-Hispanic \\
\hline Saturn & Clinical lab science & Junior & $\mathrm{F}$ & White/Black \\
\hline Tom & Human biology & Junior & M & White/non-Hispanic \\
\hline
\end{tabular}


depending on the amount of time each participant spent reasoning about a particular phenomenon. We varied the order in which phenomena were presented to ensure that all phenomena had equal representation in our data. During the interview, the interviewer provided the participant with the initial scenario for each phenomenon, then solicited the participant's explanatory ideas. The protocols are provided in the Supplemental Material.

While most of the interviewer prompts were based on what participants chose to discuss during the interview, all participants were consistently asked what they thought was happening at finer scales. This prompt was designed to encourage participants to activate conceptual resources rooted in a scientific domain that would contribute to a causal mechanistic explanation of the process they were discussing. Each participant was video-recorded during the interview and was provided a LiveScribe pen to record his or her drawings.

\section{Data Analysis}

We used a grounded theory approach to analyze our interviews in the following way: a researcher used open coding of the interview transcripts to identify the conceptual resources participants activated. We focused on how participants talked about multiscale processes and scientific ideas because of their importance in causal mechanistic explanations, although our interpretation of these ideas was shaped by the way our participants reasoned. These resources were then grouped into overarching explanatory frames that described reasoning approaches participants took when thinking about the phenomena. The researcher also evaluated instances in which participants made use of analogies to support their causal explanations. These were used to infer participants' ontological categorization of the processes they were explaining and also associated with explanatory frames depending on their characteristics. The frames and resources identified by these analyses were then discussed with the research team and revised as appropriate (Corbin and Strauss, 1990). This process was repeated until consensus was reached regarding the explanatory frames and conceptual resources based on the following criteria:

Prevalence: the explanatory frames needed to recur across multiple students' explanations and phenomena.

Reliability: the explanatory frames needed to be consistently-and independently_identifiable by individuals trained to analyze the data.

The open coding was done at the scale of discourse turns, which were determined by the interviewer prompts-when the interviewer asked the participant to either discuss a new facet of an idea the participant had brought up or to think about a current idea at a different scale, we considered that a new discourse turn for analysis. Discourse turns usually involved several exchanges between participants and the interviewer as the participants clarified and elaborated on their ideas. We excluded discourse turns that were off topic and did not directly relate to explaining our process phenomena.

For each discourse turn, we applied a code that described which frame most closely aligned with the conceptual resources the participant used regarding multiscale processes and scientific ideas. We only applied an ontological categorization code for those discourse turns in which participants invoked an analogy. In total, we coded 319 discourse turns across all interview participants, resulting in 319 multiscale processes codes and 319 scientific ideas codes. We identified 57 analogies that our participants provided across all phenomena and assigned them ontological categorization codes.

Once all discourse turns had been coded for each participant, we evaluated the total number of codes attributed to each of our explanatory frames both by a participant and within a phenomenon to elucidate 1 ) the broader patterns in reasoning that participants employed during their interviews and 2) the kinds of reasoning each of our phenomena solicited across students.

\section{RESULTS}

We present our results in three parts: first, we describe the explanatory frames that our interview participants drew upon when asked to explain complex, real-world processes and the associated conceptual resources they activated when constructing causal explanations. Second, we discuss how prevalent the different frames were in our interview participants' reasoning across all the phenomena. Finally, we use interview excerpts from representative participants to illustrate our frames and discuss the patterns of their use during the interviews.

Explanatory Frames and Associated Conceptual Resources We identified three broad explanatory frames that differentiated the kinds of explanations participants provided when explaining their understanding of our complex phenomena: a colloquial frame, an emerging mechanistic frame, and a causal mechanistic frame. Each frame characterized the different ways participants activated conceptual resources regarding multiscale processes and scientific ideas about how and why processes occur, as well as students' ontological categorization of phenomena, which are all key features of mechanistic causal explanations (Table 2). Our causal mechanistic frame is aligned with participants' abilities to construct mechanistic causal explanations, because these kinds of explanations convey a deep understanding of scientific processes.

In a colloquial explanatory frame, participants activated conceptual resources that were based on personal experience or conventional wisdom to explain the phenomena in everyday language, often assigning teleological (i.e., purpose- or goaldriven) motivations to explain why phenomena occurred. When activating conceptual resources about multiscale processes, they focused on the observable elements of the system and/or made no distinctions among elements that occur at different scales. When explaining why and/or how the phenomena occurred, the scientific ideas they used were often force-dynamic in nature and relied on actors that were intentional in their interactions or used covering law associations among the features of the system (Pinker, 2007; Braaten and Windschitl, 2011). Participants' analogies demonstrated an ontological categorization of phenomena based on surface features rather than subscale processes.

In an emerging mechanistic frame, participants used scientific ideas, principles, or relationships in their explanations in semicoherent ways, often mixing colloquial and scientific language. When activating conceptual resources about multiscale processes, they recognized elements from subscales of the system, but either made mistakes when reasoning across scales (e.g., situated molecules and cells on the same scale) or used relevant scale elements superficially (e.g., recognized a relevant molecule but did not describe how it interacted with other 
TABLE 2. The relationship between participants' conceptual resources and explanatory frames

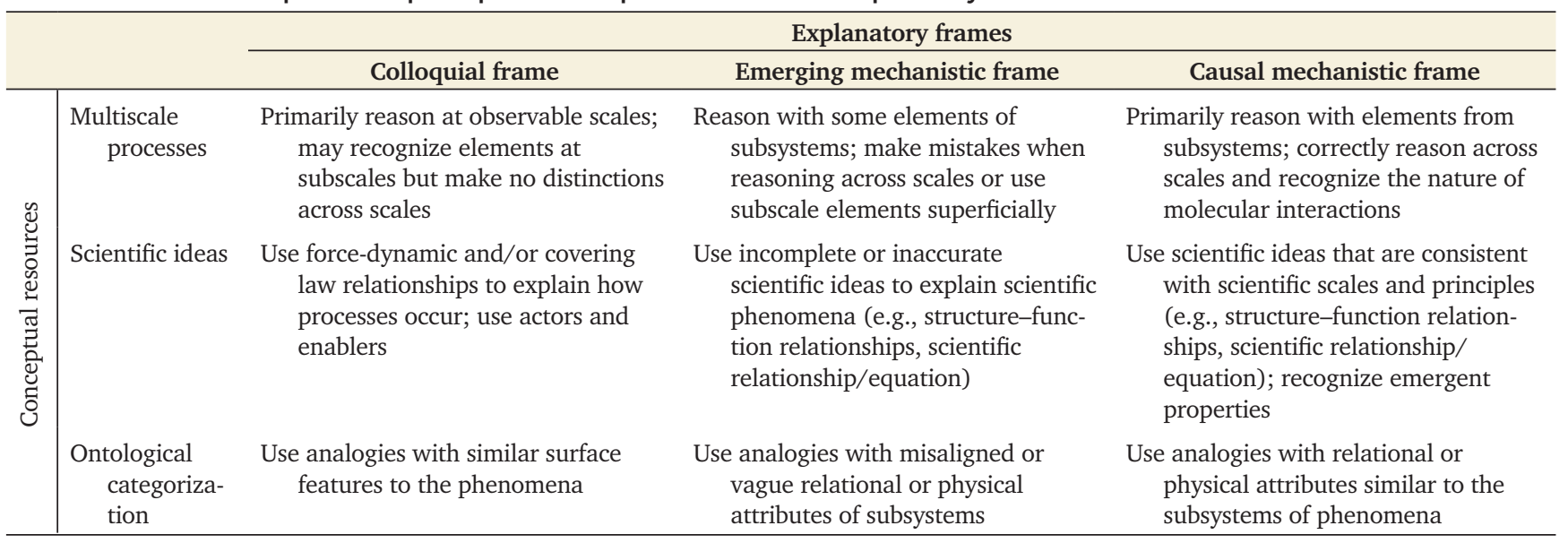

molecules or formed larger structures). Their reasons for why and/or how phenomena occurred contained scientific ideas that were incomplete or inaccurate, such as describing structure-function relationships that were partially developed or using scientific relationships or equations in inaccurate or procedural ways. Participants used ontological categorizations that compared irrelevant or incorrect subscale elements between the key phenomenon and an analogous scenario.

In a causal mechanistic frame, participants consistently and cohesively drew upon scientific conceptual resources to express their ideas in predominantly scientific language. When activating conceptual resources about multiscale processes, they recognized subscale elements of the phenomena and used them correctly to describe emergent patterns at observable scales. They drew on scientific ideas that described mechanistic reasoning constrained by scientific principles and accounted for processes that occurred at multiple scales. Their explanations usually described structure-function relationships or made use of scientific equations in productive ways. Their ontological categorizations were based on relevant subscale features between the phenomenon they were explaining and an analogous phenomenon.

\section{The Prevalence of Explanatory Frames in Student Reasoning} We systematically coded 319 total discourse turns across all student interviews and phenomena to investigate the ways participants reasoned about multiscale processes and their scientific ideas. From these analyses, we found that the colloquial frame was the most prevalent frame students invoked in their reasoning, employing it 46 and 39\% of the time when reasoning about multiscale processes and scientific ideas, respectively (Figure 1). The emerging mechanistic frame was the second most-prevalent frame, being invoked by participants 39 and $45 \%$ of the time, respectively, during their reasoning. The causal mechanistic frame was the least prevalent frame, being invoked only 15 and $8 \%$, respectively, during our participants' reasoning (see the Supplemental Material for individual participant codes). We saw a similar trend in the way participants employed analogies (total = 57 analogies), with $49 \%$ of participants drawing on analogies aligned with the colloquial frame, $47 \%$ drawing on analogies aligned with the emerging mechanistic frame, and only $4 \%$ drawing on analogies aligned with the causal mechanistic frame.
When we evaluated how participants reasoned for each phenomenon, we found a similar trend in the prevalence of each of our frames as the overall pattern, with most phenomena primarily eliciting resources aligned with the colloquial frame, with only a limited number of eliciting resources aligned with the causal mechanistic frame (Figure 2). However, there were some observable differences among phenomena. For example, the Heatburn phenomenon was overall most likely to elicit conceptual resources aligned with a causal mechanistic frame from our participants. Indeed, of the eight participants who reasoned about this phenomenon, six used conceptual resources aligned with a causal mechanistic frame at least once during their reasoning, indicating that the relatively high percentage of discourse turns containing scientifically aligned resources was due to its broad accessibility to our participants rather than capturing only one or two students who were able to speak deeply over many discourse turns about this topic. Conversely, only three of 10 participants who reasoned about the Sneeze phenomenon accessed at least one conceptual resource aligned with a causal mechanistic frame. Instead, all participants drew at least partially on colloquial-based resources to craft their

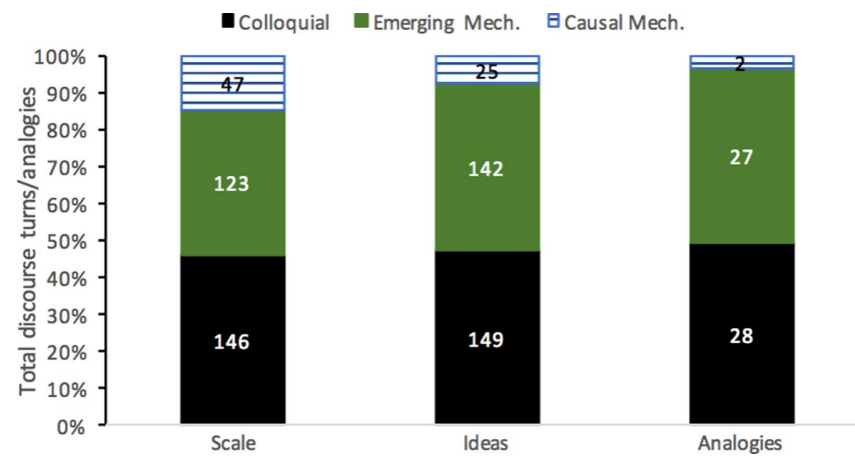

FIGURE 1. Percent of total discourse turns (319) or analogies (57) in which participants used a colloquial, emerging mechanistic, or causal mechanistic explanatory frame when invoking conceptual resources about multiscale processes ("Scale"), scientific ideas ("Ideas"), or ontological categorization ("Analogies"). The total number of discourse turns per category is provided in each bar. 


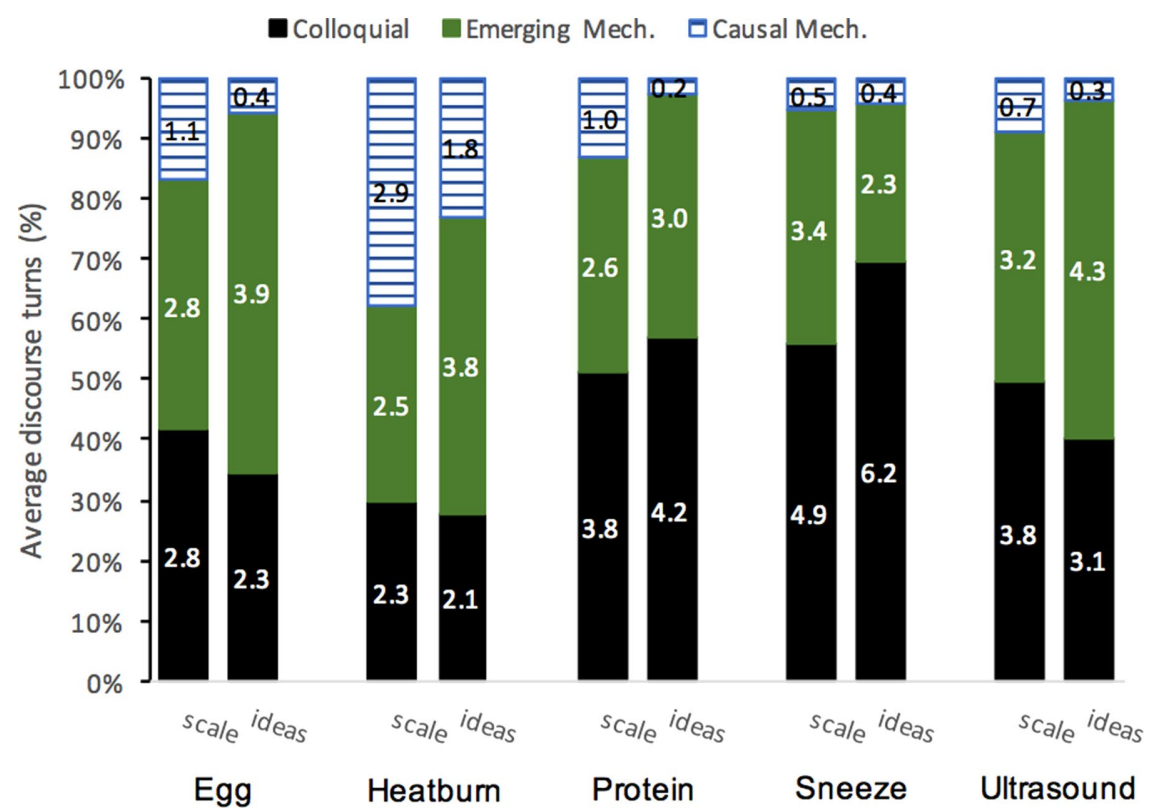

FIGURE 2. Average percent of discourse turns across all phenomena containing conceptual resources about multiscale processes ("scale") and scientific ideas ("ideas") that aligned with the frames. The average number of discourse turns per category is provided in each bar.

accounts, possibly because this topic has a well-established place in social environments with ideas about "germs," "hand-washing," and "covering your mouth when you sneeze," making these kinds of ideas easily accessible to our participants compared with detailed knowledge of virology and disease transmission. Protein also tended to elicit more colloquial rather than scientific responses.

\section{Exemplifying the Explanatory Frames and Their Conceptual Resources}

In the following sections, we use excerpts from exemplary interviews to illustrate each frame we observed our participants invoking when constructing explanations about the phenomena. The first set of excerpts depicts the ways participants invoked different types of conceptual resources about multiscale processes and scientific ideas that were emblematic of each of the explanatory frames. This set contains accounts from Saturn, Rudee, and Daniel as they reason about why a blister forms when a person touches a hot pan.

We also provide a second set of excerpts from instances when participants drew on analogies to support their explanations to demonstrate how participants' ontological categorization of processes or objects aligned with the explanatory frames. This set of excerpts contains analogies that Anna, Lisa, and Natalie activated as conceptual resources when explaining why an egg goes from runny to solid when boiled. These analogies provide insight into the ontological categorization students invoked for this phenomenon.

\section{Causal Explanations}

Saturn. We begin with an excerpt from Saturn's interview to exemplify the colloquial frame in her reasoning about how a blister forms after touching a hot pan:
Saturn: Because basically when you burnt yourself, you kill [sic] the cells in your hand. So those are dead cells now. And then your body is like oh shit, what did you just do? And it's sending different cells in there, and that's why it swells right away, and it's hot to the touch from the burn, but also from the swelling and stuff... Basically the blister forms, and it's pus and stuff because the pus is actually white blood cells and whatever trying to eat the dead cells and dissolve those, and basically get you healed up and clean up the injury. Basically, if it's bad enough that you get a blister, that means you triggered basically an immune response to get in there, and then it will blister up and it's supposed to break, so the nastiness comes out. You'll have like a very pink patch of sore skin that's brand new and eventually it'll be fine.

In this excerpt, Saturn frequently made use of colloquial language, teleological motivations, and conventional wisdom. The conceptual resources she activated around multiscale processes focused on observable objects at the macroscopic scale, with phrases like "pus and stuff," and she made little mention beyond "cells" of subscale agent interactions and how they contributed to the observable events she described.

Saturn's scientific ideas about how and why events occurred revealed her activation of conceptual resources with a narrative, sequential explanatory style. She described an initiating event (burned finger), which triggered a series of immune responses (white blood cell dissemination, blister formation), culminating in the repair of the damaged skin (Chi et al., 2012; Sevian and Talanquer, 2014). She ascribed anthropomorphic motivations for the body's response, explaining that the body is "sending different cells in there" after expressing dismay at being burned (i.e., "your body is like, 'oh shit, what did you just do?"'). She viewed white blood cells as intentional healing agents that "eat dead cells" and "basically get you healed up and clean up the injury" rather than the result of a physiological response due to a stimulus. Similarly, she described the purpose of a blister as one of healing, saying, "if it's bad enough that you get a blister, that means you triggered .... an immune response to get in there, and then it will blister up." This contrasts with the idea of physical changes in the skin that result from the input of heat. She used teleological motivations around how to treat blisters, saying, "it's supposed to break so the nastiness comes out," which is followed by the exposure of pink skin that will eventually "be fine."

Rudee. We use an excerpt from Rudee's interview to illustrate the emerging mechanistic frame, because she incorporated a number of scientific ideas into a narrative explanation for why a blister forms:

Rudee: So, touch the pan, obviously you get the reflex to move away. So you have the neurons transmitting all of the-I'm not 
going to be very scientific ... And then ... let's see. There is obviously a heat transfer. So then you have the receptors in the skin that are sensitive to heat, and then that causes the molecular change on the fingertip that results in-the first layer of the skin is damaged, and then that causes the blister to form, and that's the immune response to any sort of harm to the skin, a blister would form.

When Rudee activated conceptual resources about multiscale processes, she recognized that "molecular change" and "receptors in the skin"-both ideas about subscale elementswent together and influenced "the first layer of the skin" in a way that shows she recognized something about the emergent nature of the systems but was unclear exactly how they linked together. She drew on scientific ideas about heat transfer, heat causing molecular change, and skin layers being involved with blister formation, but she struggled to fit those pieces together in a mechanistic way. Despite the scientific ideas she began with, she reverted to teleology in her final sentence by saying, "that's the immune response to any sort of harm to the skin, a blister would form," as if the goal of the immune response was ultimately blister formation.

Daniel. We use an excerpt from Daniel's interview to illustrate the causal mechanistic frame, because he consistently relied on scientific ideas and language when constructing his explanation in ways that were mechanistic and recognized the emergent properties of the system:

Daniel: We have a stove, with the pan, and this guy touches it. We'll say he's already touched it, and the energy from the pan is going to be transferred to his hand and then to the skin cells, which will be definitely damaged from the heat. I'm going to say the proteins would be denatured, and the redness will form and the guy is going to be in a lot of pain due to the high amount of energy transferred from the pan. Yeah, I mean if there's a cellthis is the little cell. They're going to have proteins that transport membranes across, and I'm guessing that with the high amount of energy, they will be degraded, and the bonds making them up will break, and that will lead to some sort of change in how the cell looks which will result in the hand changing.

Daniel's account shows he activated conceptual resources about multiscale processes that made use of objects at subscales (i.e., proteins) and how those subscale units influenced the properties at the observable level (i.e., hand), consistent with recognizing the emergent processes. The scientific ideas he drew on directly linked the transfer of heat from the pan to the finger as the cause of the blister forming, saying, "with the high amount of energy, they [proteins] will be degraded and the bonds making them up will break, and that will ... result in the hand changing," providing a mechanism for structural change in this system. While there were aspects of Daniel's explanation that were imprecise (e.g., "proteins that transport membranes across," rather than proteins transporting other molecules across membranes), his overall explanatory approach contained scientific ideas and language consistent with someone viewing the phenomenon as a problem that could be solved with scientific tools.

Analogies. We use the following three examples of analogies participants drew on when reasoning about why an egg goes from runny to solid when it is boiled to illustrate how their explanatory frame influenced their ontological categorization of the phenomenon they were trying to explain.

Anna. Anna activated conceptual resources that likened an egg solidifying when boiled with what happens when water freezes based on their observable features in a manner consistent with an ontological categorization aligned with the colloquial frame. Consequently, she missed important differences between the two processes she compared and continued to be uncertain about her explanation. In this example, Anna and the interviewer had been discussing the nature of solids and liquids when she came up with the analogy of water freezing:

Interviewer: Okay, so there's something about the egg when it starts out and it's "looser" and then you add this heat and energy to it and it makes them become tighter together-

Anna: Solidifies them. Structured and in place, right.

Interviewer: Do you have ... an idea on why they're going from the loose to the tight, or-

Anna: Well maybe if you compare it to water freezing, you know what I mean? It'll freeze because the heat goes out or in ... I think, okay if it's freezing the heat is going out so that's exothermic? So maybe it's like an exothermic kind of thing so the particles [in the egg] are getting close together because the heat is leaving?

Her ontological grouping of eggs solidifying with water freezing into a "runny-things-become-solid" categorization resulted in Anna missing two important distinctions between these processes: 1) that eggs solidifying and water freezing have opposite relationships with the input of heat, and 2) that the mechanisms for why water and eggs solidify are distinct processes. In the first case, Anna attributed the process of "heat go[ing] out" in an "exothermic kind of thing" as the reason behind the solidification of both processes, rather than just when water freezes. It is only when the interviewer later points out that eggs solidify when heat is added that she recognized the discrepancy.

In the second case, Anna explained the solidification of the egg white as the result of "the particles ... getting close together because the heat is leaving," which is consistent with what happens when most substances (apart from water) freeze, but not when proteins - which make up eggs - denature. During protein denaturation, solidification happens when heat is added and the proteins experience conformational changes in structure that render them either less soluble than their previous form or more likely to aggregate due to newly exposed, mutually attractive functional groups (e.g., hydrophobic interactions).

Lisa. Lisa activated conceptual resources that focused on unnamed, subscale units when comparing an egg solidifying to a mixed salad, consistent with an ontological categorization aligned with our emerging mechanistic frame. Before the following excerpt, Lisa had suggested that "denaturing" may be the process causing the egg white to solidify and was mulling out loud over whether or not that process was correct by contrasting denaturing with mixing a salad: 
Lisa: Hardening. Something. You can't put it back once you boil it, and that's significant of a chemical process. Right?...

Interviewer: When you say you can't put it back, you mean like you can-

Lisa: Like you can mix up a salad and then you can separate it all into its individual parts, but you can't unboil an egg.

By using the "mixed salad" analogy, Lisa demonstrated that she recognized the egg system was composed of smaller subsystems-like the "individual parts" of a mixed salad-but unlike a mixed salad, those parts underwent a "chemical process" that could not be undone. Therefore, she recognized that the process of egg solidification was different from a phase change, unlike Anna. Lisa used the relevant subsystem features of a contrasting analogy to help her categorize eggs solidifying into a "chemical process" group, which was productive for understanding why this phenomenon occurs. However, her analogy was incomplete, because knowing that denaturing eggs was different from mixing a salad did not help her progress further toward explaining mechanistically why it was not possible to "unboil an egg."

Natalie. Natalie demonstrates how using an analogy that draws on conceptual resources focused at subscales can facilitate a deeper understanding of an unfamiliar process. This approach is consistent with an ontological categorization aligned with the causal mechanistic frame. Her reasoning is particularly interesting, because she initially focused on the observable properties of ice melting and an egg boiling in her reasoning but recognized inconsistencies at that scale that led her to re-evaluate the system at a finer scale.

Just before the excerpt below, Natalie had described that proteins were constructed of amino acids held together by covalent bonds and suggested that adding heat to the proteins resulted in "strengthening the bonds between [proteins]." The interviewer then prompted her to reason about what other things solidify when heat is added to a system.

Interviewer: Are there things other than, outside of eggs, that work kind of like that?

Natalie: When you heat it that it turns solid?

Interviewer: Sure, or maybe changes in something.

Natalie: I guess ice if you put ice into it melts. Or well, everything. Everything kind of melts but that kind of does the opposite when it is heated. Eggs kind of turn into a solid while others turn into a liquid.

Natalie initially focused on the observable properties of systems by citing ice melting as a similar process to eggs solidifying, but she quickly recognized that the water phase-change model actually described the opposite pattern to what was happening with the egg, thereby representing a contrasting example of observable properties. Later in the interview, when Natalie returned to the analogy of ice melting, she discovered a deeper relevant analogy focused on similar subsystem features between the two systems:
Natalie: Yeah, that makes sense, [proteins] start to unfold. I know that hydrogen bonds are weakest so when you add heat to that those are going to probably move first and it goes from the quaternary structure back down to its secondary or primary structure. So, you heat it even more and eventually break down the protein to its amino acids.

Interviewer: Do you have a sense of what's happening with proteins when you heat it? Like why does that help explain kind of what's going on with most things melting?

Natalie: Well yeah, because when you, say for instance you melt ice you're kind of breaking the strict hydrogen bonds between the water molecules and letting them sort of move around. You're sort of increasing the probability of your arrangements.

In this instance, she attended to the idea that disrupting the hydrogen bonds between water molecules by adding heat resulted in an increase in "the probability of your arrangements." This was a relevant feature of the melting ice subsystem that aligned with what occurs during protein denaturation.

Participants' Explanatory Frames within a Phenomenon. These excerpts demonstrate discourse turns in which participants activated conceptual resources about multiscale processes and scientific ideas consistent with one explanatory frame (e.g., colloquial frame). However, participants rarely drew exclusively on conceptual resources aligned with one explanatory frame across multiple discourse turns as they reasoned about a phenomenon. Instead, they drew from a variety of more or less sophisticated conceptual resources depending on the interviewer's prompt or their level of comfort with a particular topic.

This was realized longitudinally through the course of a participant's reasoning within a phenomenon, when the participant initially drew on resources aligned with one frame, then shifted to more or less sophisticated resources depending on the interviewer's prompts. An example of this is found in Brad's discussion about how an ultrasound produces an image of a person's interior in the Ultrasound phenomenon. He is trying to explain why an ultrasound image contains objects of different hues, saying, "I'm guessing that means like different densities of things that are inside of your body that are like that gallstone ... I don't know if there are differences like it sends-like where air is and where it's more like a solid?" He initially draws on conceptual resources about macroscopic objects consistent with a colloquial frame, such as "gallstone," "air," and "solid." When he is later asked by the interviewer whether he has any sense of what it means for something to be dense at the molecular level, he replies, "Yeah, so atoms are closer together," indicating he has resources to draw on at subscales about this topic that were aligned with our emerging mechanistic frame.

Another way we observed participants drawing on different categories of conceptual resources was when they addressed either multiscale processes or scientific ideas. For example, a participant could identify relevant subscale features in a system but then use those objects in a force-dynamic way to explain how or why those objects interacted as they did. Saturn demonstrated this scenario in the following excerpt that appeared later in her reasoning about blister formation: 
Saturn: So, happy cell with nucleus and happy little ribosomes, and endoplasmic reticulum, and other stuff because it's a cool cell. And then I suppose heat or whatever injury could lyse the cell, and then oh no, the nucleus is out over here, and he's sad because he's over here. And like all of the cytosol from inside the cell is out here, and now this cell is dead and not functioning because its insides are over here.

In this excerpt, Saturn's reasoning about multiscale processes was situated at the subscale (i.e., microscopic) level, where she identified objects such as ribosomes and endoplasmic reticulum, but the scientific ideas she used to describe how those objects interacted were force-dynamic.

Despite this variability, we observed differences in how often participants activated conceptual resources aligned with particular explanatory frames when reasoning about a phenomenon (Figure 3). For example, when we evaluated all of the conceptual resources that Saturn, Rudee, and Daniel activated about multiscale processes and scientific ideas when reasoning about blister formation, 50 and $80 \%$ of the discourse turns from Saturn, respectively, contained conceptual resources consistent with a colloquial frame, whereas Daniel drew on similar resources only 10 and $40 \%$ of the time. Instead, his reasoning was consistent with a causal mechanistic frame in 50 and $60 \%$ of the discourse turns, respectively. Rudee fell between Saturn and Daniel, having a similar number of discourse turns when she activated conceptual resources consistent with either the colloquial or emerging mechanistic frames fairly evenly. Notably, neither Saturn nor Rudee activated conceptual resources aligned with a causal mechanistic frame for this phenomenon despite being prompted to reason at subscales and pressed to further explain their ideas by the interviewer.

Participants' Explanatory Frames across Phenomena. We also observed differences in participants' overall approaches to reasoning when we evaluated the conceptual resources that they accessed when explaining multiple phenomena. For example, Natalie drew primarily on conceptual resources aligned with a causal mechanistic frame for all the phenomena she was explaining except Ultrasound, which she only briefly discussed (Figure 4). Conversely, Julie drew similarly from conceptual resources aligned with colloquial and emerging mechanistic frames for all phenomena, only once activating an accurate and appropriate scientific conceptual resource. Her lack of access to resources associated with a causal mechanistic frame is notable considering she averaged almost twice as many discourse turns per phenomenon compared with Natalie, meaning Julie experienced roughly twice as many prompts from the interviewer to delve deeper into her reasoning and access more sophisticated resources. Her inability to do this suggests that these kinds of conceptual resources were either more difficult-or possibly not available-for Julie to draw on during her reasoning of this phenomenon. However, Natalie's ability to make use of science-based conceptual resources with less prompting from the interviewer suggests she could more easily, and readily, access a causal mechanistic frame to facilitate her reasoning.

We also observed that some students were more likely to access conceptual resources aligned with a causal mechanistic frame for only certain phenomena. For example, Daniel activated a number of conceptual resources associated with a causal mechanistic frame when reasoning about the Heatburn phenomenon, but drew heavily on resources aligned with a colloquial frame when explaining the Sneeze and Ultrasound phenomena (Figure 4C).

\section{DISCUSSION}

In this study, we asked undergraduate science majors to explain complex, real-world phenomena to better understand how the students constructed causal explanations about scenarios found outside their science classes. We focused on causal mechanistic explanations, because they express a deep understanding of the scientific processes that explain how and why phenomena occur (Braaten and Windschitl, 2011; NRC, 2012b; Weinrich and Talanquer, 2015). When participants in this study employed a causal mechanistic frame, they were demonstrating a facility with causal mechanistic explanations that helped them reason productively about novel phenomena, even when their explanations were not canonically correct. However, we found that the causal mechanistic frame was the least prevalent frame invoked by our participants despite all of them being declared science majors several semesters into their degree programs. Instead, participants frequently drew on more colloquial ideas and rationales for why events occurred (i.e., colloquial frame), consistent with observations made by others studying undergraduate students (e.g., the "cognitive construals" of Coley and Tanner, 2012, 2015; Weinrich and Talanquer, 2015; Southard et al., 2017).

It is important to note here that we interviewed only 12 students who volunteered for our study, so it is quite possible that we would encounter a higher

FIGURE 3. Percent of discourse turns containing conceptual resources about multiscale processes ("Scale") and scientific ideas ("Ideas") that aligned with a particular frame for Saturn, Rudee, and Daniel when explaining blister formation after touching a hot pan. The number of discourse turns per category is provided in each bar. 

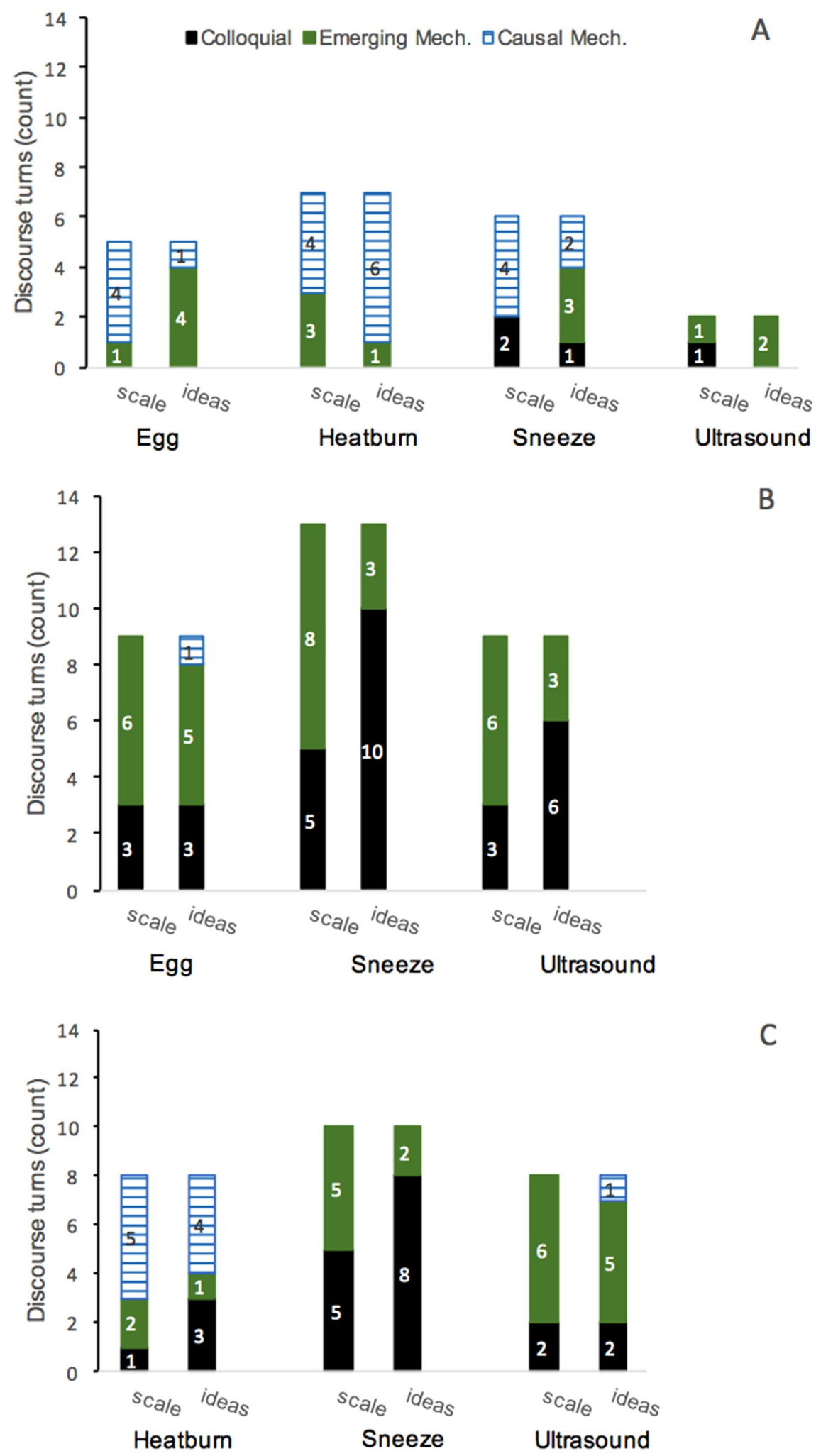

FIGURE 4. Number of discourse turns across all phenomena for Natalie (A), Julie (B), and Daniel (C) containing conceptual resources about multiscale processes ("scale") and scientific ideas ("ideas") that aligned with the frames. The number of discourse turns per category is provided in each bar. frequency of students consistently reasoning with a causal mechanistic frame in a broader population. However, in a companion study to this one, which administered an online survey version of the interview phenomena to undergraduates in physics, chemistry, and biology courses at two large institutions, we observed a similar pattern. Preliminary results show that, when answering one item (i.e., a Sneeze assessment item), only $4 \%$ of students (36 out of 806) drew on conceptual resources aligned with a causal mechanistic frame when reasoning about multiscale processes. This suggests that the pattern we observed with our interview data is not exclusive to our small population.

B

\section{How Are Students Limited by Not Reasoning Mechanistically?}

The relatively low frequency of interview participants employing a causal mechanistic frame indicates that many of the participants struggled to leverage the knowledge that they had been exposed to during their course work to reason about complex, realworld phenomena in a mechanistic way. This was made evident by the prevalent use of the emerging mechanistic frame by many of our interview participants, who struggled to 1) identify appropriate scientific concepts to explain the phenomena and 2) apply scientific concepts to real-world scenarios. These struggles often arose from their tendency to focus on more surface-level features of the phenomena rather than subsystem features (e.g., molecules) or processes. Consequently, they often selected scientific concepts that had only limited explanatory power and led them along unproductive lines of reasoning. This contrasts with participants who invoked a causal mechanistic frame, whose focus on subsystem features and processes helped them identify and use scientific concepts that were mechanistic in nature and provide more comprehensive explanation of the phenomena. We see the difficulty of students who invoke an emerging mechanistic frame to identify and apply relevant scientific concepts to novel phenomena as a critical limitation for students who do not draw on mechanistic conceptual resources during their reasoning. Below, we describe in more detail how this limits students' ability to identify and apply relevant scientific concepts to explain real-world phenomena. 
When students focus on more surface-level features and processes, they can develop ontological categorization schemes that link fundamentally dissimilar processes together in ways that lead them to apply scientific concepts with limited relevance to novel phenomena (Chi et al., 1994). This is at the root of the first struggle-identifying appropriate scientific concepts-that participants drawing on an emerging mechanistic frame experienced. We observed this when Lisa used a mixed salad analogy to help her explain what happens to an egg when it is boiled. We also observed this when several participants likened an ultrasound to a radar or x-ray. While ultrasound, radar, and x-ray all share the general characteristics of waves, like frequency, reflection, and refraction, their lack of similarity in subscale object attributes (i.e., ultrasound uses mechanical waves, radar and $\mathrm{x}$-rays use electromagnetic waves) contributed to participants' difficulty in explaining how ultrasounds actually worked.

Focusing on surface-level features and processes also constrains students' ability to apply scientific concepts to real-world phenomena. We observed this during Rudee's reasoning about the physiological processes that occur to form a blister after touching a hot pan, where she identified several subscale elements and structures but was unclear how to link them together. We saw this again in another instance when Anna tried to apply the equation for Gibbs free energy to explain what happens to an egg when it is boiled. While she could identify all the variables in the equation, she had difficulty linking them to the egg system she was trying to explain, because she focused on surface relationships like "temperature change" and "reaction." Using the Gibbs free energy equation in this scenario is potentially quite productive-there is actually an increase in entropy when egg white becomes solid, contrary to what typically happens when many liquids solidify. However, this equation is a covering law explanation and therefore not inherently mechanistic (Braaten and Windschitl, 2011). To further investigate the unusual increase in entropy, students would need to incorporate ideas about molecules (i.e., proteins), such as the way they are arranged in space and how they respond to changes in temperature, which are concepts that support mechanistic reasoning. Therefore, without this mechanistic component, students may be limited in how deeply they can understand phenomena regardless of how well they can describe relationships among variables.

\section{How Can Educators Help Students Develop Mechanistic Reasoning?}

Currently, many university science courses are structured to privilege breadth of content over depth of coverage (AAAS, 2011; Cooper et al., 2015) in ways that can result in recontextualizing science into a discipline that is fact rich but experience poor, the very opposite of how science itself is practiced (Sharma and Anderson, 2009; Gunckel, 2010). While this is an efficient way to convey and explain a broad swath of scientific concepts, it often deprives students of rich opportunities to reason about how those concepts work in the complex, real-world environments that scientists seek to understand.

Therefore, to help students improve their facility with constructing causal mechanistic explanations, we advocate for incorporating phenomenological reasoning opportunities into undergraduate science curricula. This will provide students with experience in selecting and integrating scientific concepts as they use those concepts in real-world settings (Jacobson and
Wilensky, 2006; Southard et al., 2017). This approach builds on the changing focus of science education curricula and instruction toward three-dimensional reasoning around phenomena rather than disciplinary knowledge alone (AAAS, 2011; NRC, 2012b). We suggest the following recommendations to help guide instructors in implementing phenomenological reasoning opportunities in their courses to support students in developing and refining causal mechanistic reasoning:

1. Narrow the number of concepts that students must learn to a few key principles that can be used to explain multiple processes (Cooper et al., 2017). Focusing instruction on core ideas can help limit the number of concepts that students must sort through to find relevant ideas for explaining phenomena-a particular challenge for students who use an emerging mechanistic frame. It can also help students develop a coherent understanding of how processes across systems are related. Modell's (2000) general models approach to understanding physiological phenomena provides a practical example of this by showing how phenomena in multiple systems of the human body follow the same guiding principles.

2. Use phenomena to reveal core ideas of science (Windschitl et al., 2012; Reiser, 2013). For example, Wilson et al. (2006) and Jin and Anderson (2012) use phenomena such as plant growth, animal movement, and decay to introduce carbon-transforming processes like photosynthesis and cellular respiration and to reveal how the principles of matter or energy conservation can be used to guide students' development of rigorous mechanistic explanations for these phenomena. Using phenomena to help students identify core scientific ideas can provide them with a powerful reasoning framework that they can use when considering novel phenomena. This may be especially productive for students who reason with an emerging mechanistic frame by helping them focus on key ideas amid the variety of sophisticated scientific ideas they already have.

3. Encourage generative mechanistic reasoning. Generative mechanistic reasoning occurs when students develop explanations that are plausibly aligned with concepts in a domain, even if they contain inaccuracies (Southard et al., 2017). This approach encourages students to draw on their extant ideas rather than factual recall alone and can facilitate deep reasoning opportunities. Regular practice constructing generative mechanistic explanations may help students learn to see the explanatory potential of the ideas they are exposed to in their courses and target mechanistic ideas.

4. Provide epistemic tools that scaffold causal mechanistic reasoning about phenomena. Epistemic tools are practical or conceptual scaffolds that operate "between an individual and the accomplishment of a complex task that might otherwise be out of reach without some form of assistance" and support scientific thinking (Windschitl et al., 2012, p. 887). Multiple research groups at the K-12 level are investigating ways to develop epistemic tools centered around phenomena that help instructors scaffold student learning toward sophisticated scientific practice. ${ }^{1}$ These efforts recognize

${ }^{1}$ See the following resources: Tools for Ambitious Science Teaching University of Washington (n.d.); Activate Learning (2012); Anderson et al. (2018); NextGen: Next Generation Science Exemplar (2018). 
that students benefit from formal guidance when explaining phenomena using a scientific epistemology (Windschitl et al., 2012). Therefore, developing and using epistemic tools that guide students in developing explanations that account for the multiscale nature of systems and are guided by scientific ideas can help students progress toward proficiency in reasoning with a causal mechanistic frame.

Incorporating learning opportunities based on phenomena into undergraduate courses can help students achieve the aspirations set out in Vision and Change (AAAS, 2011), which recognized that "future scientists and nonscientists alike must become adept at making connections among seemingly disparate pieces of information, concepts, and questions" (p. 3). It is critical for educators to help students develop a scientific epistemology that allows them to cohesively activate relevant conceptual resources. Only in this way can they successfully tackle the complex socioeconomic and environmental issues facing society today. Our work provides an important step toward characterizing how students approach complex processes and can help guide the development of explicit instructional methods that equip students with the skills needed to productively reason about complex processes they encounter in their personal and professional lives.

\section{ACKNOWLEDGMENTS}

Funding for this work was provided by Michigan State University (though a CREATE for STEM Institute LPF-CMP 2 Innovation Grant, STEM Gateway Fellowship award, and Lyman Briggs College) and Florida International University (through State of Florida UP:LIFT project and start-up funding). We thank Abigail Sundberg and Dr. Angie Little for helping with the project.

\section{REFERENCES}

Activate Learning. (2012). IQWST (Investigating and Questioning our World through Science and Technology). Retrieved May 9, 2018, from activatelearning.com/iqwst.

American Association for the Advancement of Science. (1993). Benchmarks for science literacy. New York: Oxford University Press

American Association for the Advancement of Science. (2011). Vision and change in undergraduate biology education: A call to action. Washington, DC.

Anderson, C. W., de los Santos, E. X., Bodbyl-Roels, S., Covitt, B., Edwards, K. D., Hancock, J. B., ... Welch, M. (2018). Designing educational systems to support enactment of the Next Generation Science Standards. Journal of Research in Science Teaching, https://doi.org/10.1002/tea.21484

Berland, L. K., \& Hammer, D. (2012). Students' framings and their participation in scientific argumentation. In Khine, M. S. (Ed.), Perspectives on scientific argumentation (pp. 73-93). Dordrecht, Netherlands: Springer. https:// doi.org/10.1007/978-94-007-2470-9_5

Braaten, M., \& Windschitl, M. (2011). Working toward a stronger conceptualization of scientific explanation for science education. Science Education, 95(4), 639-669. https://doi.org/10.1002/sce.20449

Carey, S. (1986). Cognitive science and science education. American Psychologist, 41(10), 1123

Chi, M. T. (2013). Two kinds and four sub-types of misconceived knowledge, ways to change it, and the learning outcomes. In Vosniadou, S. (Ed.), International handbook of research on conceptual change (2nd ed., pp. 49-70). New York: Routledge.

Chi, M. T. H., Feltovich, P., \& Glaser, R. (1981). Categorization and representation of physics problems by experts and novices. Cognitive Science, 5 , $121-152$
Chi, M. T. H., Roscoe, R. D., Slotta, J. D., Roy, M., \& Chase, C. C. (2012). Mis conceived causal explanations for emergent processes. Cognitive Science, 36(1), 1-61. https://doi.org/10.1111/j.1551-6709.2011.01207.x

Chi, M. T. H., Slotta, J. D., \& De Leeuw, N. (1994). From things to processes: A theory of conceptual change for learning science concepts. Learning and Instruction, 4(1), 27-43. https://doi.org/10.1016/0959 -4752(94)90017-5

Clark, D., \& Linn, M. C. (2003). Designing for knowledge integration: The impact of instruction time. Journal of Learning Sciences, 12(4), 451-493.

Coley, J. D., \& Tanner, K. D. (2012). Common origins of diverse misconceptions: Cognitive principles and the development of biology thinking CBE-Life Sciences Education, 11(3), 209-215.

Coley, J. D., \& Tanner, K. (2015). Relations between Intuitive biological thinking and biological misconceptions in biology majors and nonmajors. CBE-Life Sciences Education, 14(1), ar8.

Cooper, M. M., Caballero, M. D., Ebert-May, D., Fata-Hartley, C. L., Jardeleza, S. E., Krajcik, J. S., ... Underwood, S. M. (2015). Challenge faculty to transform STEM learning. Science, 350(6258), 281-282. https://doi .org/10.1126/science.aab0933

Cooper, M. M., Corley, L. M., \& Underwood, S. M. (2013). An investigation of college chemistry students' understanding of structure-property relationships. Journal of Research in Science Teaching, 50(6) $699-721$.

Cooper, M. M., Kouyoumdjian, H., \& Underwood, S. M. (2016). Investigating students' reasoning about acid-base reactions. Journal of Chemical Education, 93, 1703-1712.

Cooper, M. M., Posey, L. A., \& Underwood, S. M. (2017). Core ideas and topics: Building up or drilling down? Journal of Chemical Education, 94 541-548.

Corbin, J. M., \& Strauss, A. (1990). Grounded theory research: Procedures, canons, and evaluative criteria. Qualitative Sociology, 131, 3-21.

Gentner, D. (1989). The mechanisms of analogical learning. In Vosniadou, S. \& Ortony, A. (Eds.), Similarity and analogical reasoning (pp. 199-241). New York: Cambridge University Press.

Ginsburg, H. (1997). Entering the child's mind: The clinical interview in psychological research and practice. New York: Cambridge University Press.

Gunckel, K. L. (2010). Experiences, patterns, and explanations. Science and Children, 48(1), 46

Gupta, A., Hammer, D., \& Redish, E. F. (2010). The case for dynamic models of learners' ontologies in physics. Journal of the Learning Sciences, 19(3), 285-321. https://doi.org/10.1080/10508406.2010.491751

Hammer, D., \& Elby, A. (2002). On the form of a personal epistemology. In Hofer, B. K., \& Pintrich, P. R. (Eds.), Personal epistemology: The psychology of beliefs about knowledge and knowing (pp. 169-190). Mahwah NJ: Erlbaum

Hammer, D., Elby, A., Scherr, R. E., \& Redish, E. F. (2005). Resources, framing, and transfer. In Mestre, J. P. (Ed.), Transfer of learning from a modern multidisciplinary perspective (pp. 89-119). Greenwich, CT: IAP.

Hesse, J. J., \& Anderson, C. W. (1992). Students' conceptions of chemical change. Journal of Research in Science Teaching, 29(3), 277-299.

Hmelo-Silver, C. E., \& Azevedo, R. (2006). Understanding complex systems: Some core challenges. Journal of the Learning Sciences, 15(1), $53-61$

Jacobson, M. J., \& Wilensky, U. (2006). Complex systems in education: Scientific and educational importance and implications for the learning sciences. Journal of the Learning Sciences, 15(1), 11-34.

Jin, H., \& Anderson, C. W. (2012). A learning progression for energy in socio-ecological systems. Journal of Research in Science Teaching, 49(9) 1149-1180. https://doi.org/10.1002/tea.21051

Keil, F. (2012). Does folk science develop? In Shrager, J., \& Carver, S. (Eds.) The journey from child to scientist: Integrating cognitive development and the education sciences (pp. 67-86). Washington, DC: American Psychological Association.

Ledford, H. (2015). Team science. Nature, 525, 308-311.

Lombrozo, T., \& Vasilyeva, N. (2017). Causal explanation. In Waldmann, M (Ed.), Oxford handbook of causal reasoning (pp. 415-432). Oxford, UK: Oxford University Press. 
Maeyer, J., \& Talanquer, V. (2010). The role of intuitive heuristics in students thinking: Ranking chemical substances. Science Education, 94(6), 963984. https://doi.org/10.1002/sce.20397

McNeill, K. L., \& Krajcik, J. (2008). Inquiry and scientific explanations: Helping students use evidence and reasoning. In Luft, J., Bell, R. L., \& Gess-Newsome, J. (Eds.), Science as inquiry in the secondary setting (121-134). National Science Teachers Association-NSTA Press.

Modell, H. I. (2000). How to help students understand. Advanced Physiology Education, 23, 101-107.

Mohan, L., Chen, J., \& Anderson, C. W. (2009). Developing a multi-year learning progression for carbon cycling in socio-ecological systems. Journal of Research in Science Teaching, 46(6), 675-698. https://doi .org/10.1002/tea.20314

Mozzer, N. B., \& Justi, R. (2012). Students' pre- and post-teaching analogical reasoning when they draw their analogies. International Journal of Science Education, 34(3), 429-458. https://doi.org/10.1080/09500693. 2011.593202

National Research Council. (2012a). Discipline-based education research: Understanding and improving learning in undergraduate science and engineering. Washington, DC: National Academies Press.

National Research Council. (2012b). A framework for $K-12$ science education: Practices, crosscutting concepts, and core ideas. Washington, DC National Academies Press.

NextGen: Next Generation Science Exemplar. (2018). NGSX: Next Generation Science Exemplar. Retrieved May 9, 2018, from www.ngsx.org.

Passmore, C., Gouvea, J. S., \& Giere, R. (2013). Models in science and in learning science: Focusing scientific practice on sense-making. In Matthews, M. R. (Ed.), International handbook of research in history, philosophy and science teaching (pp. 1171-1202). Dordrecht: Springer Netherlands. http://doi.org/10.1007/978-94-007-7654-8_36

Pinker, S. (2007). The stuff of thought: Language as a window into human nature. New York: Viking.

Redish, E. F. (2004). A theoretical framework for physics education research: Modeling student thinking. ArXiv Preprint Physics/0411149. Retrieved October 12, 2017, from https://arxiv.org/abs/physics/0411149

Reiser, B. J. (2013, September). What professional development strategies are needed for successful implementation of the Next Generation Science Standards? Paper presented at: the Invitational Research Symposium on Science Assessment ( $K-12$ Center at ETS).

Rubin, H. J., \& Rubin, I. (2005). Qualitative interviewing (2nd ed.). Thousand Oaks, CA: Sage.

Russ, R., Coffey, J., Hammer, D., \& Hutchison, P. (2009). Making classroom assessment more accountable to scientific reasoning: A case for attending to mechanistic reasoning. Science Education, 93(5), 875-891.

Russ, R. S., Lee, V. R., \& Sherin, B. L. (2012). Framing in cognitive clinical interviews about intuitive science knowledge: Dynamic student understandings of the discourse interaction. Science Education, 96(4), 573-599. https://doi.org/10.1002/sce.21014
Russ, R. S., Scherr, R. E., Hammer, D., \& Mikeska, J. (2008). Recognizing mechanistic reasoning in student scientific inquiry: A framework for discourse analysis developed from philosophy of science. Science Education, 92(3), 499-525.

Scherr, R. E., \& Hammer, D. (2009). Student behavior and epistemological framing: Examples from collaborative active-learning activities in physics. Cognition and Instruction, 27(2), 147-174. https://doi.org/10.1080/ 07370000902797379

Sevian, H., \& Talanquer, V. (2014). Rethinking chemistry: A learning progression on chemical thinking. Chemistry Education Research and Practice. 15(1), 10-23. https://doi.org/10.1039/C3RP00111C

Sharma, A., \& Anderson, C. W. (2009). Recontextualization of science from lab to school: Implications for science literacy. Science and Education, 18(9), 1253-1275. https://doi.org/10.1007/s11191-007-9112-8

Shtulman, A., \& Valcarcel, J. (2012). Scientific knowledge suppresses but does not supplant earlier intuitions. Cognition, 124, 209-215.

Southard, K. M., Espindola, M. R., Zaepfel, S. D., \& Bolger, M. S. (2017). Generative mechanistic explanation building in undergraduate molecular and cellular biology. International Journal of Science Education, 39(13), 1795-1829.

Stevens, R., Wineburg, S., Herrenkohl, L. R., \& Bell, P. (2005). Comparative understanding of school subjects: Past, present, and future. Review of Educational Research, 75, 125-157.

Talmy, L. (1988). Force dynamics in language and cognition. Cognitive Science, 12, 49-100.

University of Washington (n.d.). Tools for Ambitious Science Teaching. Retrieved May 9, 2018, from ambitiousscienceteaching.org.

Vosniadou, S. (1989). Analogical reasoning as a mechanism in knowledge acquisition: A developmental perspective. In Vosniadou, S., \& Ortony, A. (Eds.), Similarity and analogical reasoning (pp. 413-437). New York: Cambridge University Press.

Vosniadou, S., \& Ortony, A. (1989). Similarity and analogical reasoning: A synthesis. In Vosniadou, S., \& Ortony, A. (Eds.), Similarity and analogical reasoning (pp. 1-17). New York: Cambridge University Press.

Weinrich, M. L., \& Talanquer, V. (2015). Mapping students' conceptual modes when thinking about chemical reactions used to make a desired product. Chemical Education Research and Practice, 16(3), 561-577. https://do .org/10.1039/C5RP00024F

Wilson, C. D., Anderson, C. W., Heidemann, M., Merrill, J. E., Merritt, B. W., Richmond, G., ... Parker, J. M. (2006). Assessing students' ability to trace matter in dynamic systems in cell biology. Cell Biology Education, 5(4), $323-331$.

Windschitl, M., Thompson, J., \& Braaten, M. (2008). Beyond the scientific method: Model-based inquiry as a new paradigm of preference for school science investigations. Science Education, 92(5), 941-967.

Windschitl, M., Thompson, J., Braaten, M., \& Stroupe, D. (2012). Proposing a core set of instructional practices and tools for teachers of science. Science Education, 96(5), 878-903. 